\title{
Fraccionamiento e interesterificacion del aceite de palma (Elaeis guineensis) cultivado en la amazonia peruana
}

\author{
Por Dora García de Sotero', Jorge Sandoval del Águila1, Robinson Saldaña Ramírez", \\ Gladys Cárdenas de Reátegui ${ }^{1}$, José Antonio Soplín Ríos ${ }^{1}$, Víctor Sotero Solís ${ }^{2}$, \\ Rosángela Pavan Torres ${ }^{3}$ y Jorge Mancini Filho ${ }^{3}$
}

\author{
${ }^{1}$ Facultad de Ingeniería Química, Iquitos-Perú. Universidad Nacional de la Amazonía Peruana. \\ ${ }^{2}$ Instituto de Investigaciones de la Amazonia Peruana. \\ ${ }^{3}$ Faculdade de Ciências Farmacêuticas, Universidade de São Paulo São Paulo-SP-Brasil. \\ Mail de contacto: dora@usp.br
}

\section{RESUMEN}

Fraccionamiento e interesterificacion del aceite de palma (Elaeis guineensis) cultivado en la amazonia peruana.

En el presente trabajo se realizó el estudio de las características físicas y químicas del fruto de la palma aceitera procedente de la cuenca del Manití. (Región Loreto - Perú). Del mismo modo se realizó el fraccionamiento e interesterificación de las mezclas de aceite de palma y estearina en las proporciones. Sobre el aceite crudo y los productos se determinaron las propiedades físico-químicos y análisis de ácidos grasos mediante la cromatografía gaseosa. El aceite de palma presenta una concentración de ácido grasos saturados de $51,17 \%$ y cuando fraccionado a $25^{\circ} \mathrm{C}$, este se incrementa en la estearina a $54,31 \%$. Los mejores productos para la industria de alimentos son las mezclas interesterificadas de estearina tanto sola como con sus mezclas con aceite de palma, dado que presentan puntos de fusión próximos a $37^{\circ} \mathrm{C}$.

PALABRAS-CLAVE: Aceite de palma - Elaeis guineensis - Fraccionamiento - Mezclas - Interesterificación.

\section{SUMMARY}

Fractionation and interesterification of palm (Elaeis guineensis) oil from Peruvian Amazon

In the present work, the physical and chemical characteristics of the fruit of the oily palm coming from the river basin of the Manití (Region Loreto - Peru) were studied. Also, the fractionation of the palm oil and the interesterification of mixtures of palm oil/estearin was carried out. Physico- chemical properties of the crude oil and of the products obtained and fatty acids were analysed by gas chromatography. The level of saturated fatty acids increased from $51,17 \%$ in the palm oil to $54,31 \%$ in the stearin. The best products for the food industry were the interesterified samples as they had melting points close to $37^{\circ} \mathrm{C}$.

KEY-WORDS: Elaeis guineensis - Fractionation Interesterification - Mixtures - Palm oil.

\section{INTRODUCCIÓN}

La palma aceitera (Elaeis guineenssis) es una palmera originaria de los trópicos del África, cuyo fruto da un aceite de optima calidad, con alta concentración de ácidos palmítico y oleico y que con las técnicas de modificación adecuadas permite obtener una amplia variedad de productos para la industria de alimentos, entre los cuales se encuentran los aceites de cocina, mantecas, bases para margarinas, "vanaspati" (grasa vegetal sustituto de la mantequilla en la cocina) y, para la industria oleoquímica, materias primas para la fabricación de jabón, jabones metálicos, velas, y grasas lubricantes (Berger y Ong,1985; PORIM, 1988; Boza,1990). Esta palmera se encuentra actualmente siendo cultivada en las regiones de San Martín y Loreto, e incluso el estado peruano ha declarado su cultivo de interés nacional (Perú, 2000).

La siembra de palma aceitera en la cuenca del Maniti (Región Loreto-Perú) se inició en el año de 1981, con la firma del convenio entre ORDELORETO (Organismo de desarrollo de Loreto) y la empresa EMDEPALMA (Empresa para el desarrollo y explotación de palma aceitera), ubicando y desarrollando los viveros y parcelas en el caserío de Santa Cecilia.

El fraccionamiento del aceite es considerado como un proceso de modificación termomecánico, donde los triglicéridos componentes del aceite son separados generalmente como una mezcla de cristalización parcial, en una fase líquida (oleina) y otra sólida (estearina) (Thomas III, 1985). Se han desarrollado diferentes métodos para el fraccionamiento de aceites, principalmente con el objetivo de modificar su textura y punto de fusión. Esta técnica permite aislar las diversas clases de triglicéridos sin cambiar la distribución de la posición de los ácidos grasos y su utilización en la industria se da en el caso de obtener sucedáneos de la manteca de cacao, obtención de aceites láuricos a partir del aceite de coco y fraccionamiento de la grasa de leche (Willis et al., 1998).

La mezcla de aceites y grasas permite obtener productos finales con características propias. Ello podría ser considerado como un método de modificación de aceites y grasas, sobre todo debido a las 
interacciones que tienen lugar entre los triglicéridos que promueven alteraciones en las propiedades físicas de los productos oleosos (Smalwood, 1989; Birker y Padley, 1987).

La interesterificación es un proceso que modifica la distribución natural de los ácidos grasos en las moléculas de los triglicéridos. La composición de los ácidos grasos permanece idéntica a la grasa - mezcla original, pero las propiedades físicas, como punto de fusión, contenido de grasa sólida y forma de cristalización, son modificadas (Gioielli, 1996, Sonntang, 1982).

La interesterificación puede realizarse por simple calentamiento de los aceites y grasas a temperaturas que bordean los $300^{\circ} \mathrm{C}$, pero la reacción a esta condición es lenta y normalmente acompañada de descomposición y polimerización de los triglicéridos. En la industria se utilizan catalizadores para disminuir la temperatura de la reacción. Los catalizadores más usados son los metales alcalinos y sus derivados, siendo el metóxido de sodio el más empleado, debido a sus ventajas de costo, manipulación y temperatura de reacción, que puede ser reducida al rango de $30-90{ }^{\circ} \mathrm{C}$ (Gioielli, 1996; Hurtová et al., 1996).

El objetivo del presente trabajo fue el de realizar los análisis físicos y químicos del fruto, así como efectuar la modificación del aceite de palma mediante el fraccionamiento, mezclas e interesterificación con los frutos procedentes de la cuenca del Maniti-Región Loreto-Perú.

\section{PARTE EXPERIMENTAL}

\subsection{Muestras y tratamientos}

Se utilizaron frutos de palma aceitera (Elaeis guineensis) recolectados de los sectores Paparo y Paparillo, en el Distrito de Indiana, Provincia de Maynas, Región Loreto, distante a unos $80 \mathrm{Km}$ de la ciudad de lquitos, zona de la Cuenca del Maniti.

Los frutos fueron llevados a una pequeña planta extractora en la misma zona de recolección y primero se sometieron a cocción por aproximadamente tres horas y posteriormente, se extrajo el aceite con una prensa manual. Este aceite fue sometido a:

a) Fraccionamiento: El aceite se colocó en cinco vasos de precipitado de $100 \mathrm{~mL}$ y se colocaron en baños a temperaturas de 15 , 20,25 y $30^{\circ} \mathrm{C}$ por tres horas. De inmediato los productos fueron filtrados a temperatura ambiente, obteniéndose las fracciones de oleica (parte liquida) y estearina (parte sólida).

b) Mezclas: Se realizaron mezclas binarias del aceite de palma con su estearina en diferentes proporciones de acuerdo al diseño experimental indicado en la Tabla 1.

c) Interesterificación: Las mezclas se sometieron a la reacción de interesterificación, para lo cual se utiliza como catalizador el metoxido de sodio en la concentración de
Tabla 1

Diseño experimental de las mezclas de aceite de palma y de su estearina

\begin{tabular}{ccc}
\hline $\begin{array}{c}\text { Número de } \\
\text { muestra }\end{array}$ & $\begin{array}{c}\text { Proporción de } \\
\text { aceite de palma } \mathbf{x}_{\mathbf{1}}\end{array}$ & $\begin{array}{c}\text { Proporción de } \\
\text { estearina } \mathbf{x}_{\mathbf{2}}\end{array}$ \\
\hline 1 & 1 & 0 \\
2 & 0 & 1 \\
3 & $2 / 3$ & $1 / 3$ \\
4 & $1 / 3$ & $2 / 3$ \\
\hline
\end{tabular}

$0,75 \%$ en peso con respecto al peso de la mezcla, de acuerdo a Sotero-Solis et al. (2001).

\subsection{Determinaciones analíticas}

Los análisis de composición proximal se realizo según las Normas del Instituto Adolfo Lutz (1976), que incluyó los análisis de humedad, proteína, aceite y fibra. La concentración de carbohidratos fueron obtenidos por la diferencia de 100 menos la suma de los porcentajes de agua, proteína, aceite y ceniza. Para la caracterización de los aceites se determino el punto de fusión, densidad, índices de refracción, de acidez y de yodo, según los métodos de la AOAC (1995). La concentración de $\beta$-caroteno por el método espectrofotmetrico, según Higby (1962). La composición de ácidos grasos se determinó de acuerdo al método de Hartman y Lago (1973).

Para el análisis de composición de los ácidos grasos se utilizó el cromatógrafo de gas marca Shimadzu, modelo CG17A, utilizando una columna capilar de sílica fundida VA-WAX (polieteilenglicol) de $30 \mathrm{~m}$ de largo y $0,25 \mathrm{~mm}$ de diámetros interior, el gas de arrastre fue helio con flujo de $1 \mathrm{~mL} / \mathrm{minu}$ to. La programación de temperatura en la columna fue isotérmica a $150{ }^{\circ} \mathrm{C}$ por 11 minutos y la velocidad de calentamiento $3{ }^{\circ} \mathrm{C} / \mathrm{min}$. hasta $210^{\circ} \mathrm{C}$, permaneciendo por 5 minutos, siendo que la temperatura de vaporización fue de $250^{\circ} \mathrm{C}$ y la del detector de $280^{\circ} \mathrm{C}$.

\section{RESULTADOS Y DISCUSIÓN}

En la Tabla 2, se presenta las características físicas del fruto así como su análisis proximal. El tamaño y peso del fruto es de $4,22 \mathrm{~cm}$ y $14.29 \mathrm{~g}$ con muy poca variación entre la población muestreada. El peso de la pulpa y semilla con relación al fruto es de $72,36 \%$ y $27,71 \%$ respectivamente. Estos datos corresponden a la variedad denominada "Dura" según Boza (1990) y que a pesar de no haber mantenido un adecuado manejo, aún conserva las características propias de esta. Asimismo, se observa que la concentración de aceite en la pulpa es de $69,21 \%$ lo que representa en el fruto la cantidad de $50 \%$ de aceite en fruto, un poco superior al obtenido por la empresa Palma del Espino del Perú (Hidalgo, 2005), quienes reportan $45 \%$. 
Tabla 2

Características físicas y composición proximal del fruto de la palma aceitera (Elaeis guineensis), del Maniti

\begin{tabular}{lr}
\hline \multicolumn{1}{c}{ Características físicas } & \multicolumn{1}{c}{ Media } \\
\hline Longitud del fruto $(\mathrm{cm})$ & $4,22 \pm 0,50$ \\
Diámetro del fruto $(\mathrm{cm})$ & $2,76 \pm 0,30$ \\
Peso del fruto $(\mathrm{g})$ & $14,29 \pm 3,77$ \\
Peso de la pulpa $(\mathrm{g})$ & $10,31 \pm 2,65$ \\
Longitud de la semilla $(\mathrm{cm})$ & $3,04 \pm 0,34$ \\
Diámetro de la semilla $(\mathrm{cm})$ & $1,79 \pm 0,31$ \\
Peso de la semilla $(\mathrm{g})$ & $3,96 \pm 1,31$ \\
\multicolumn{1}{c}{ Composición proximal } & \\
Humedad, g/100g & \\
Ceniza, g/100g & $13,08 \pm 0,09$ \\
Aceite, g/100g & $0,26 \pm 0,02$ \\
Proteína, g/100g & $69,21 \pm 0,11$ \\
Fibra, g/100g & $1,86 \pm 0,01$ \\
Carbohidratos, g/100g & $4,56 \pm 0,01$ \\
\hline
\end{tabular}

La Tabla 3 muestra los ensayos y el rendimientote de oleina y estearina obtenidos tras el fraccionamiento en seco del aceite de palma a cuatro temperaturas diferentes, se observa que a $25{ }^{\circ} \mathrm{C}$ se obtiene un buen fraccionamiento, esta temperatura difiere con la reportada por Thomas III (1985) quienes indican que el fraccionamiento lo realizaron a $10{ }^{\circ} \mathrm{C}$ y $20{ }^{\circ} \mathrm{C}$. Esta diferencia se debe, posiblemente, a que ellos trabajaron el fraccionamiento con solvente.

En la Tabla 4, se presentan las características físico-químicas del aceite de palma y sus productos de fraccionamiento, así como la concentración de caroteno y retinol de los mismos, se observa que
Tabla 3

Ensayos del fraccionamiento del aceite de palma a diferentes temperaturas

\begin{tabular}{|c|c|c|c|}
\hline Temperatura $\left({ }^{\circ} \mathbf{C}\right)$ & Estearina (\%) & \multicolumn{2}{|c|}{ Oleina (\%) } \\
\hline 15 & 100,00 & \multicolumn{2}{|r|}{0,00} \\
\hline 20 & 100,00 & \multicolumn{2}{|r|}{0,00} \\
\hline 25 & 78,75 & \multicolumn{2}{|r|}{21,25} \\
\hline 30 & 76,00 & \multicolumn{2}{|r|}{24,00} \\
\hline \multicolumn{4}{|c|}{$\begin{array}{c}\text { Tabla } 4 \\
\begin{array}{c}\text { Características físico químicas, } \beta \text {-caroteno y reti- } \\
\text { nol del aceite de palma y de los productos } \\
\text { del fraccionamiento }\end{array}\end{array}$} \\
\hline Determinaciones & $\begin{array}{c}\text { Aceite } \\
\text { de palma }\end{array}$ & Oleina & Estearina \\
\hline Punto de fusión, ${ }^{\circ} \mathrm{C}$ & 13,50 & - & 28,00 \\
\hline Densidad, $\mathrm{g} / \mathrm{mL}$ & 0,9151 & 0,9165 & 0,9019 \\
\hline $\begin{array}{l}\text { Índice de refracción } \\
\text { Índice de }\end{array}$ & 1,4605 & 1,4660 & 1,4629 \\
\hline saponificación & 204.35 & 203.44 & 204,66 \\
\hline Índice de lodo & 50.48 & 54.34 & 46,86 \\
\hline$\beta$-Caroteno, mg/100g & 0,359 & 0,355 & 0,361 \\
\hline Retinol, mg/100g & 2,520 & 2,529 & 2,613 \\
\hline
\end{tabular}

comparando a la estearina con el aceite original esta incrementa notablemente su punto de fusión, alcanzando $28^{\circ} \mathrm{C}$, así como una ligera disminución de la densidad y la mejora en la concentración de $\beta$-caroteno y retinol. Como era de esperar la estearina presenta un menor índice de iodo debido al menor número de instauraciones en las moléculas de sus ácidos grasos.

Observando la Tabla 5, donde se reporta la concentración de ácidos grasos para el aceite de

Tabla 5

Composición de ácidos grasos del aceite de palma y de los productos del fraccionamiento

\begin{tabular}{|c|c|c|c|}
\hline Ácido graso & Aceite de Palma & Oleína & Estearina \\
\hline $12: 0$ & $0,05 \pm 0,02$ & $0,05 \pm 0,01$ & $0,03 \pm 0,02$ \\
\hline $14: 0$ & $1,14 \pm 0,09$ & $1,13 \pm 0,05$ & $1,19 \pm 0,06$ \\
\hline $15: 0$ & $0,03 \pm 0,02$ & $0,03 \pm 0,02$ & $0,05 \pm 0,01$ \\
\hline $16: 0$ & $45,24 \pm 0,13$ & $41,86 \pm 0,11$ & $48,22 \pm 0,07$ \\
\hline $16: 1$ & $0,19 \pm 0,03$ & $0,20 \pm 0,01$ & $0,17 \pm 0,01$ \\
\hline $17: 0$ & $0,13 \pm 0,05$ & $0,09 \pm 0,00$ & $0,10 \pm 0,01$ \\
\hline $18: 0$ & $4,13 \pm 0,10$ & $3,91 \pm 0,06$ & $4,24 \pm 0,04$ \\
\hline $18: 1(n-9)$ & $36,50 \pm 0,48$ & $39,51 \pm 0,17$ & $34,72 \pm 0,22$ \\
\hline $18: 1(n-7)$ & $0,73 \pm 0,01$ & $0,80 \pm 0,02$ & $0,69 \pm 0,01$ \\
\hline $18: 2(n-6)$ & $10,60 \pm 0,46$ & $11,30 \pm 0,16$ & $9,44 \pm 0,07$ \\
\hline $18: 3(n-3)$ & $0,28 \pm 0,02$ & $0,30 \pm 0,01$ & $0,25 \pm 0,01$ \\
\hline $20: 0$ & $0,36 \pm 0,01$ & $0,35 \pm 0,01$ & $0,36 \pm 0,01$ \\
\hline $20: 1(n-9)$ & $0,12 \pm 0,02$ & $0,12 \pm 0,01$ & $0,10 \pm 0,01$ \\
\hline $22: 0$ & $0,04 \pm 0,04$ & $0,06 \pm 0,01$ & $0,06 \pm 0,01$ \\
\hline $22: 1(n-9)$ & $<0,04$ & $<0,03$ & $<0,03$ \\
\hline $22: 2(n-6)$ & $0,19 \pm 0,01$ & $0,20 \pm 0,01$ & $0,21 \pm 0,03$ \\
\hline $24: 0$ & $0,05 \pm 0,04$ & $0,06 \pm 0,00$ & $0,07 \pm 0,01$ \\
\hline $\mathrm{n}, \mathrm{i}$ & $0,22 \pm 0,15$ & $0,03 \pm 0,09$ & $0,10 \pm 0,03$ \\
\hline Saturados & $51,17 \pm 0,18$ & $47,54 \pm 0,15$ & $54,31 \pm 0,09$ \\
\hline Monoinsaturados & $37,54 \pm 0,43$ & $40,62 \pm 0,15$ & $35,68 \pm 0,23$ \\
\hline Poliinsaturados & $11,08 \pm 0,46$ & $11,80 \pm 0,17$ & $9,90 \pm 0,11$ \\
\hline
\end{tabular}

n,i: no identificados. 
palma, oleina y estearina, obtenidos por cromatografía gaseosa, se puede observar que la concentración de ácidos grasos del aceite crudo de palma, no difiere de forma importante de los indicados por Berger y Ong (1985), quienes realizaron análisis al aceite de palma procedente de Malasia, destacando siempre la presencia de ácido palmítico $(45,24 \%)$, oleico $(36,50 \%)$ y linoléico $(10,60 \%)$. Asimismo la estearina presenta una mayor concentración de ácidos grasos saturados $(54,31 \%)$ que el aceite crudo de palma $(51,17 \%)$ y su oleina $(47,54 \%)$.

De acuerdo a la Tabla 6, donde se reporta las propiedades físico-químicas de los productos antes y después de la interesterificación, no se observa grandes diferencias en los índices de yodo y saponificación, así como con la densidad e índice de refracción. Esto se debe a que en las reacciones de interesterificación, solamente hay un rearreglo molecular de los ácidos grasos en torno de la molécula de glicerol (Gioielli, 1996; Sonntang, 1982). En cuanto a las variaciones de los puntos de fusión antes y después de la interesterificación, concuerdan con lo afirmado por Sonntang (1982), quien indica que en muestras de bajo punto de fusión este aumenta después de la interesterificación; lo cual también fue reportado por Sotero-Solis et al. (2001) al trabajar con mezclas del aceite de castaña y su grasa hidrogenada. De este modo se observa que la estearina de palma interesterificada, así como las proporciones de mezcla de estearina y sus mezclas con aceite de palma, mantienen un punto de fusión muy próximo al de la temperatura del cuerpo humano, lo cual los vuelven productos interesantes para la industria alimenticia en cuanto serviría para la preparación de margarinas, helados y productos de panificación. Según los resultados de los análisis de $\beta$-caroteno y retinol, se observa que no hubo degradación de estos compuestos cuando fue sometido a la reacción de interesterificación. Las concentraciones de B-carotenos guardan concordancia con los obtenidos por Choo et al. (1993) quienes efectuaron análisis de aceite de palma procedente de Malasia.
En la Tabla 7 se presenta la composición de ácidos grasos de las mezclas antes de ser interesterificadas y en la Tabla 8 después de esta reacción. No se observa mayor diferencia en la composición de estos compuestos al comparar los productos iniciales con los finales, debido a que solo se realizó un intercambio intermolecular de los esteres de los ácidos grasos (Sonntang, 1982) y como se indicó anteriormente, los cambios son específicamente en las propiedades físicas; aunque se observa un ligero incremento en los ácidos grasos saturados de las muestras 3 y 4 , posiblemente debido al decremento de algunos ácidos grasos mono y poliinsaturados en la reacción de interesterificación.

\section{CONCLUSIONES}

1. Con el aceite crudo obtenido en la cuenca del Maniti, Región Loreto-Perú, el mejor fraccionamiento se lleva a cabo a $25^{\circ} \mathrm{C}$.

2. Los análisis de caracterización del aceite de palma, oleina y estearina no difieren de los presentados en los trabajos realizados con la misma materia prima en Malasia.

3. Con las mezclas de estearina con el aceite de palma interesterificadas, en sus diferentes proporciones, se obtienen productos que muy bien se pueden utilizar en la industria de alimentos, sea en la preparación de margarinas, helados o industria de panificación.

4. La presencia de $\beta$-caroteno indican una excelente concentración de estos importantes compuestos, útiles tanto como vitamina así como antioxidantes.

\section{AGRADECIMIENTOS}

Al Departamento de Bromatología y Nutrición Experimental de la Facultad de Farmacia de la Universidad de São Paulo, por el apoyo a este trabajo.

Tabla 6

Características físico-químicas, $\beta$-caroteno y retinol de las mezclas antes y después de la interesterificación

\begin{tabular}{|c|c|c|c|c|c|c|c|c|}
\hline \multirow{3}{*}{ Determinaciones } & \multicolumn{8}{|c|}{ Muestras } \\
\hline & \multicolumn{4}{|c|}{ Sin Interesterificar } & \multicolumn{4}{|c|}{ Interesterificadas } \\
\hline & 1 & 2 & 3 & 4 & 1 & 2 & 3 & 4 \\
\hline Punto de fusión & 13,50 & 28,00 & 21,33 & 19,50 & 43,17 & 38,33 & 40,00 & 36,83 \\
\hline Densidad & 0,9151 & 0,9019 & 0,9010 & 0,9021 & 0,9192 & 0,9134 & 0,9057 & 0,9072 \\
\hline Índice de refracción & 1,4605 & 1,4629 & 1,4628 & 1,4641 & 1,4630 & 1,4630 & 1,4651 & 1,4646 \\
\hline Índice de saponificación & 204,35 & 204,66 & 204,05 & 204,10 & 203,84 & 203,09 & 203,19 & 203,18 \\
\hline Índice de lodo & 50,48 & 46,86 & 46,99 & 47,98 & 53,47 & 47,90 & 47,86 & 47,82 \\
\hline$\beta$-Caroteno, mg/100g & 0,359 & 0,361 & 0,362 & 0,366 & 0,356 & 0,368 & 0,353 & 0,390 \\
\hline Retinol, mg/100g & 2.520 & 2.613 & 2.560 & 2.580 & 2.515 & 2.624 & 2.509 & 2.585 \\
\hline
\end{tabular}


Tabla 7

Composición de ácidos grasos de las mezclas antes de la interesterificación

\begin{tabular}{ccccc}
\hline Ácido graso & \multicolumn{4}{c}{ Muestras } \\
\cline { 2 - 5 } $12: 0$ & $0,05 \pm 0,01$ & $0,03 \pm 0,02$ & $\mathbf{3}$ & $0,08 \pm 0,01$ \\
$14: 0$ & $1,13 \pm 0,05$ & $1,19 \pm 0,06$ & $1,14 \pm 0,09$ & $1,33 \pm 0,08$ \\
$15: 0$ & $0,03 \pm 0,02$ & $0,05 \pm 0,01$ & $0,03 \pm 0,02$ & $<0,08$ \\
$16: 0$ & $41,86 \pm 0,11$ & $48,22 \pm 0,07$ & $45,24 \pm 0,13$ & $42,84 \pm 0,20$ \\
$16: 1$ & $0,20 \pm 0,01$ & $0,17 \pm 0,01$ & $0,19 \pm 0,03$ & $0,19 \pm 0,01$ \\
$17: 0$ & $0,09 \pm 0,00$ & $0,10 \pm 0,01$ & $0,13 \pm 0,05$ & $0,12 \pm 0,02$ \\
$18: 0$ & $3,91 \pm 0,06$ & $4,24 \pm 0,04$ & $4,13 \pm 0,10$ & $3,95 \pm 0,12$ \\
$18: 1$ (n-9) & $39,51 \pm 0,17$ & $34,72 \pm 0,22$ & $36,50 \pm 0,48$ & $38,31 \pm 0,39$ \\
$18: 1$ (n-7) & $0,80 \pm 0,02$ & $0,69 \pm 0,01$ & $0,73 \pm 0,01$ & $0,73 \pm 0,01$ \\
$18: 2$ (n-6) & $11,30 \pm 0,16$ & $9,44 \pm 0,07$ & $10,60 \pm 0,46$ & $11,35 \pm 0,23$ \\
$18: 3$ (n-3) & $0,30 \pm 0,01$ & $0,25 \pm 0,01$ & $0,28 \pm 0,02$ & $0,33 \pm 0,02$ \\
$20: 0$ & $0,35 \pm 0,01$ & $0,36 \pm 0,01$ & $0,36 \pm 0,01$ & $0,35 \pm 0,01$ \\
$20: 1$ (n-9) & $0,12 \pm 0,01$ & $0,10 \pm 0,01$ & $0,12 \pm 0,02$ & $0,12 \pm 0,02$ \\
$22: 0$ & $0,06 \pm 0,01$ & $0,06 \pm 0,01$ & $0,04 \pm 0,04$ & $<0,08$ \\
$22: 1$ (n-9) & $<0,03$ & $<0,03$ & $<0,04$ & $<0,08$ \\
$22: 2$ (n-6) & $0,20 \pm 0,01$ & $0,21 \pm 0,03$ & $0,19 \pm 0,01$ & $0,17 \pm 0,01$ \\
$24: 0$ & $0,06 \pm 0,00$ & $0,07 \pm 0,01$ & $0,05 \pm 0,04$ & $<0,08$ \\
n,i, & $0,03 \pm 0,09$ & $0,10 \pm 0,03$ & $0,22 \pm 0,15$ & $0,13 \pm 0,00$ \\
Saturados & $47,54 \pm 0,15$ & $54,31 \pm 0,09$ & $51,17 \pm 0,18$ & $48,66 \pm 0,17$ \\
Monoinsaturados & $40,62 \pm 0,15$ & $35,68 \pm 0,23$ & $37,54 \pm 0,43$ & $39,35 \pm 0,37$ \\
Poliinsaturados & $11,80 \pm 0,17$ & $9,90 \pm 0,11$ & $11,08 \pm 0,46$ & $11,85 \pm 0,23$ \\
\hline
\end{tabular}

n,i: no identificados

Tabla 8

Composición de ácidos grasos de las mezclas después de la interesterificación

\begin{tabular}{|c|c|c|c|c|}
\hline \multirow{2}{*}{ Ácido graso } & \multicolumn{4}{|c|}{ Muestras } \\
\hline & 1 & 2 & 3 & 4 \\
\hline $12: 0$ & 0,08 & - & - & - \\
\hline $14: 0$ & 1,33 & $1,08 \pm 0,00$ & $1,09 \pm 0,01$ & $1,11 \pm 0,01$ \\
\hline $15: 0$ & $<0,08$ & - & - & $0,03 \pm 0,02$ \\
\hline $16: 0$ & 42,84 & $47,92 \pm 0,33$ & $47,91 \pm 0,19$ & $47,03 \pm 0,01$ \\
\hline $16: 1$ & $0,19 \pm$ & $0,16 \pm 0,00$ & $0,15 \pm 0,00$ & $0,15 \pm 0,01$ \\
\hline $17: 0$ & $0,12 \pm 0,02$ & $0,10 \pm 0,01$ & $0,09 \pm 0,01$ & $0,09 \pm 0,01$ \\
\hline $18: 0$ & $3,95 \pm 0,12$ & $4,22 \pm 0,01$ & $4,33 \pm 0,02$ & $4,41 \pm 0,01$ \\
\hline $18: 1(n-9)$ & $38,31 \pm 0,39$ & $35,59 \pm 0,26$ & $35,33 \pm 0,13$ & $35,68 \pm 0,04$ \\
\hline $18: 1(n-7)$ & $0,73 \pm 0,01$ & $0,61 \pm 0,01$ & $0,58 \pm 0,02$ & $0,57 \pm 0,00$ \\
\hline $18: 2(n-6)$ & $11,35 \pm 0,23$ & $9,62 \pm 0,08$ & $9,74 \pm 0,04$ & $10,11 \pm 0,02$ \\
\hline $18: 3(n-3)$ & $0,33 \pm 0,02$ & $0,24 \pm 0,00$ & $0,23 \pm 0,01$ & $0,24 \pm 0,01$ \\
\hline $20: 0$ & $0,35 \pm 0,01$ & $0,36 \pm 0,00$ & $0,37 \pm 0,00$ & $0,38 \pm 0,00$ \\
\hline $20: 1(n-9)$ & $0,12 \pm 0,02$ & $0,11 \pm 0,01$ & $0,11 \pm 0,00$ & $0,11 \pm 0,00$ \\
\hline $22: 0$ & $<0,08$ & - & $0,07 \pm 0,01$ & $0,07 \pm 0,00$ \\
\hline $22: 1(n-9)$ & $<0,08$ & - & - & $0,03 \pm 0,02$ \\
\hline $22: 2(n-6)$ & $0,17 \pm 0,01$ & - & - & - \\
\hline $24: 0$ & $<0,08$ & - & - & - \\
\hline n.i. & $0,13 \pm 0,00$ & - & - & - \\
\hline Saturados & $48,66 \pm 0,17$ & $53,67 \pm 0,33$ & $53,86 \pm 0,20$ & $53,12 \pm 0,04$ \\
\hline Monoinsaturados & $39,35 \pm 0,37$ & $36,48 \pm 0,26$ & $36,17 \pm 0,15$ & $36,53 \pm 0,03$ \\
\hline Poliinsaturados & $11,85 \pm 0,23$ & $9,86 \pm 0,08$ & $9,98 \pm 0,05$ & $10,35 \pm 0,02$ \\
\hline
\end{tabular}

n.i: no identificados 


\section{BIBLIOGRAFÍA}

Association of Official Analytical Chemists. 1995. Official methods of analysis of the Association of Official Analysis Chemists. 16ed. v. 2, AOAC, Washington.

Berger K, Ong S.1985. The industrial uses of palm and coconut oils. Oleagineux 40, 613-621.

Birker P, Padley F. 1987. Physical properties of fats and oils en R.J. Hamilton y Bhali A. P. (Eds) Recent advances in Chemistry and Technology 1-11. Elsevier London.

Boza A. 1990. Cultivo de la palma africana de aceite. Banco Agrario. Lima.

Choo YM, Ma AN, Yap SC, Ooi CK, Basron Y. 1993 Production and applications of deacidified and deodorized red palm oil. Palm oil developmnets 19, 30-34.

Gioielli LA. 1996. Óleos y gorduras vegetais composição e tecnologia., Rev. Bras. Farmacognosia 5, 211-232.

Hartman L, Lago RCA. 1973. Rapid preparation of fatty acid methyl ester from lipids. Lab. Pract. 22, 475477.

Hidalgo C. 2005. Buenas Nuevas del Huallaga., Caretas: Suplemento Especial contratado. Mayo 26, Lima.

Higby WK. 1962. A simplified method for determination of the carotenoid distribution in natural and carotene fortified orange juice. J., Food Sci, 27, 42-49.

Hurtová S, Schmidt S, Zemanovic J, Simon P, Skretar S. 1996. Random interesterification of fat blends with alkali catalysts. Fett lipids 98, 60-65.
Instituto Adolfo Lutz.1976. Normas analíticas do Instituto Adolfo Lutz: Métodos químicos e físicos para analise de alimentos, 2ed., São Paulo.

Perú 2000. Decreto supremo Nº15-2000-AG., Declaran de interés nacional la instalación de plantaciones de palma aceitera. El Peruano Mayo 07, Lima.

PORIM (Palm Oil Research Institute of Malaysia). 1988. Minilibro de los usos del aceite de palma, Ed., Percetekan, Malindo, Malasia.

Smalwood NJ. 1989. Using computers for oil blending. J., Am., Oil Chem Soc. 66, 644-648.

Sonntang NOV. 1982. Fat splitting esterification and interesterification, en Bailey'slndustrial Oil and Fat

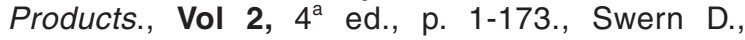
(Ed).,Wiley - Interscience, New York.,

Sotero-SolisVE, Gioielli LA, Polackiewickz B. 2001. Hidrogenación e interesterificación del aceite de castaña de Brasil (Bertholletia excelsa). Grasas y Aceites 52, 192-197.

Thomas III AE. 1985. Fractionation and winterization: Processes and products Bailey's Industrial Oil and Fat Products, Vol. 3, $4^{\mathrm{a}}$ ed, p. 1-39., en Applewhite T.,H (Ed) John Wiley \& Sons New York.

Willis WM, Lencki RW, Marangoni AG. 1998. Lipid modification strategies in the production of nutritionally functional fats and oils. Crit. Rev. Food Sci. Nutr. 38, 630674

Recibido: 28/03/07 Aceptado: 22/10/07 